\title{
THE APPLICATION OF FRACTIONAL ZENER MODEL ON MCL
}

\author{
Nada Santrač and Jasmina Pavkov, \\ Faculty of Medicine \\ University of Novi Sad, Serbia \\ nadasantrac@hotmail.com
}

\begin{abstract}
On the basis of recently published experimental results of Abramowitch et al. (2004), dealing with viscoelastic properties of sham operated and healing MCL, we show that the fractional Zener model of viscoelastic body seems to be very tractable tool for rheological description of different states of biological tissues. In this paper medial collateral ligament of the knee will be described by means of the method of Dankuc et al. 2010, who examined middle ear structures and ramp-and hold stress relaxation experiments. Two different states of MCL were described by four different constants representing modulus of elasticity, the order of fractional derivative and two relaxation constants. Predictions of the model are in good agreement with the experimental results.
\end{abstract}

Keywords: medial collateral ligament biomechanics, ramp-and-hold stress relaxation

\section{Introduction}

Injuries of knee ligaments are very common. Some estimations showed that the incidence could be 2/1000 people every year in the general population and for those involved in sports it has a much higher rate (Woo, Abramovitch, Kilger, \& Liang, 2006).

About $90 \%$ of knee ligament injuries involve the anterior cruciate ligament (ACL) and the medial collateral ligament (MCL) /Woo et al., 2006/. After rupture MCL can heal spontaneously. However, laboratory studies have shown that healing MCL ligament substance has the mechanical properties which remain considerably inferior to those of normal ligaments even after years of remodeling (Abramowitch, Woo, Clineff, \& Debski, 2004). After a few weeks of immobilization of rabbit hind limbs decreases in the structural properties of the femur-MCL-tibia complex were

\footnotetext{
* Corresponding author. Faculty of Medicine, University of Novi Sad, Hajduk Veljkova 3, 21000 Novi Sad, Serbia, e-mail:jasmina.pavkov.ns@gmail.com

(C) 2013 Faculty of Sport and Physical Education, University of Novi Sad, Serbia
} 
observed. 9 weeks of immobilization required up to one year of remobilization to reverse those changes and to return ligament properties to normal levels.

Water constitutes about 65 to $70 \%$ of ligament's total weight. The major constituent which is primarily responsible for ligament's tensile strength is Type I collagen. Another fibrous protein found in ligaments is elastin and its role is to increase flexibility. Other major components are collagen Type III and Type V (Woo et al., 2006).

Ligaments are three-dimensional anisotropic structures. Also, ligament substance have properties of solid materials as well as fluid materials thus is denoted as viscoelastic material.The complex interaction of collagen with elastin, proteoglycans, ground substance and water results in the time- and history-dependent viscoelastic behaviors of ligaments (Woo et al., 2006; Grahovac and Zigic, 2010).

There are such medical conditions which can change soft tissue remodeling due to degenerative processes, infectious or mechanic lesions. Those conditions have huge impact to the tissue function and adaptive response to the traumatic conditions (Dankuc, Kovincic, \& Spasic, 2010).

Due the interaction between adaptation of the tissue and the mechanical condition within tissue is very complex, for studying this interrelation mathematical models are needed. These models are mainly studied within biomechanics. One of the first definitions of biomechanics - mechanics applied to biology - should be replaced with the one that highlights development, extension and application of mechanics for better understanding of physiology and pathophysiology, but also the diagnosis and treatment of disease and injury (Dankuc et al., 2010).

Ligaments exhibit viscoelastic properties that depend on their state. Bodies that show creep, stress-relaxation, and hysteresis like behavior in force displacement diagram and memory effects are called viscoelastic bodies. Therefore viscoelastic properties of the ligaments can be described by nonlinear models, the standard linear viscoelastic model (so called the Zener model), or the standard fractional viscoelastic model called the fractional Zener model (see Spasic \& Charalambakis, 2002). By use of the lastone we shall describe viscoelastic properties of MCL. Namely, the fractional Zener model is able to predict behavior of the viscoelastic material with significant accuracy, including only four parameters, which is the main advantage when compared to other possibilities, no matter how complex material is considered.

Figure 1 shows the system under consideration and the position of the ligament to be modeled.

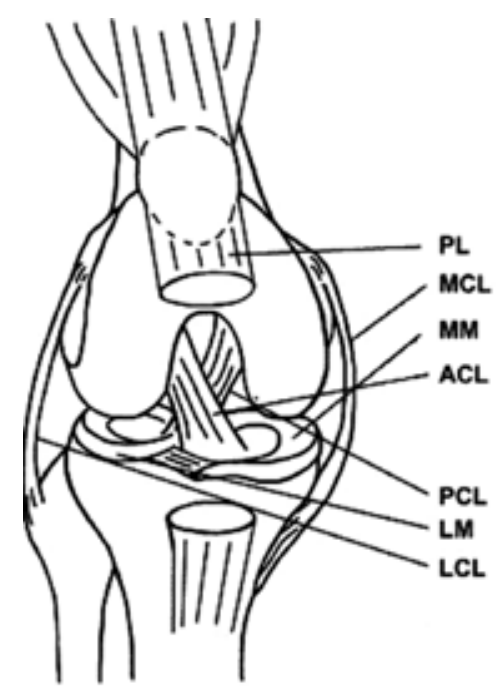

Figure 1. System under consideration 
We recognize the knee structure where PL stands for patellar ligament, MCL - medial collateral ligament, $\mathrm{MM}$ - medial meniscus, ACL - anterior cruciate ligament, PCL - posterior cruciate ligament, LM - lateral meniscus, LCL - lateral collateral ligament.

In what follows we shall put the results of the ramp-and-hold strain type of stress-relaxation experiments of Abramowitch et al. (2004), in the framework of fractional model suggested by Dankuc et al. (2010).

\section{The model (methods)}

For a body in a deformed state, the Hooke law states that stress (force per unit area) and strain can be related by

$$
\sigma=\bar{E} \varepsilon \text {, }
$$

where $\sigma$ stands for the stress at time $t, \bar{E}>0$ is the constant called modulus of elasticity, and $\varepsilon$ stands for the strain at time $t$. The standard viscoelastic body or the Zener model, states the stress-strain relation in the following form:

$$
\sigma+\tau_{\sigma 1} \sigma^{(1)}=\bar{E}_{1} \varepsilon+\bar{E}_{1} \tau_{\varepsilon 1} \varepsilon^{(1)}
$$

with $\tau_{\sigma 1}$ as a constant known as stress relaxation time, $\bar{E}_{1}$ is modulus of elasticity and $\tau_{\varepsilon 1}$ denotes strain relaxation time constant. Here $(\cdot)^{(1)}=\frac{d}{d t}(\bullet)$ stands for the first derivative with respect to time $t$. The second law of thermodynamics and the stability conditions implies that in (2) the following restrictions on the constants must be satisfied

$$
\bar{E}_{1}>0, \quad \tau_{\sigma 1}>0, \quad \tau_{\varepsilon 1}>\tau_{\sigma 1}
$$

In order to describe specific class of viscoelastic materials we introduce $\alpha$-the derivative, with $0<\alpha<1$, of a function $u(t)$ in the Riemann-Liouville form, and replace the first derivative in (2) by the one of order $\alpha$, say:

$$
\frac{d^{\alpha}}{d t^{\alpha}} u(t)=u^{(\alpha)} \equiv \frac{d}{d t} \frac{1}{\Gamma(1-\alpha)} \int_{0}^{t} \frac{u(\xi) d \xi}{(t-\xi)^{\alpha}}
$$

where $\Gamma$ is the Euler gamma function. In such a way we obtain the fractional derivative type generalization of (2), usually called a fractional Zener body, (see Bagely \& Torvik, 1986):

$$
\sigma+\tau_{\sigma \alpha} \sigma^{(\alpha)}=E_{\alpha} \varepsilon+E_{\alpha} \tau_{\varepsilon \alpha} \varepsilon^{(\alpha)}
$$

where $0<\alpha<1$, and $\tau_{\sigma \alpha}, \tau_{\varepsilon \alpha}$ and $E_{\alpha}$ are constants. The dimension of $\tau_{\sigma \alpha}$ and $\tau_{\varepsilon \alpha}$ is time to the power of $\alpha$.

The constitutive equation (4) describes uniaxial, isothermal deformation of the viscoelastic body of negligible mass, together with fundamental restrictions on the coefficients of the model, that follow from the Clausius-Duhem inequality (Bagely \& Torvik, 1986; Atanackovic, 2002):

$$
E_{\alpha}>0, \tau_{\sigma \alpha}>0, \tau_{\varepsilon \alpha}>\tau_{\sigma \alpha}
$$


For examining the ramp-and-hold strain then stress relaxation experiment, the preparation should be done by applying the Laplace transform, see Dankuc et al. (2010), to the following strain function

$$
\varepsilon(t)=\left\{\begin{array}{c}
\kappa t, \text { for } 0 \leq t \leq t_{k}, \\
\vdots: \vdots \\
\varepsilon_{0}=\kappa t_{k}=\text { const., for } t \geq t_{k}
\end{array}\right.
$$

where $\kappa=$ const. stands for the initial strain rate, and $t_{k}$, represent the time instant when the prescribed strain is achieved.

Following the lines of Dankuc et al. (2010.) one gets the following form for the stress in the viscoelastic tissue after applying strain as in eq. (6):

$$
\sigma(t)=\left\{\begin{array}{c}
E_{\alpha} \kappa t+\mu \kappa \int_{0}^{t} e_{\alpha}(\xi, \lambda) d \xi, \text { for } 0<t \leq t_{k} \\
\vdots: \vdots \\
E_{\alpha} \kappa t_{k}+\mu \kappa\left[\int_{0}^{t} e_{\alpha}(\xi, \lambda) d \xi-\int_{9}^{t-t_{k}} e_{\alpha}(\xi, \lambda) d \xi\right], \text { for } t>t_{k}
\end{array}\right.
$$

where $e_{\alpha}(t ; \lambda)$ stands for the Mittag-Leffler function, and where $\lambda$ and $\mu$ denotes $1 / \tau_{\sigma \alpha}$ and $E_{\alpha}\left(\tau_{\varepsilon \alpha} / \tau_{\sigma \alpha}-1\right)$ respectively. The deformation pattern given in form (6), (7) is known as the real stimulus, since almost all real materials exhibit that type of behavior, see Figure

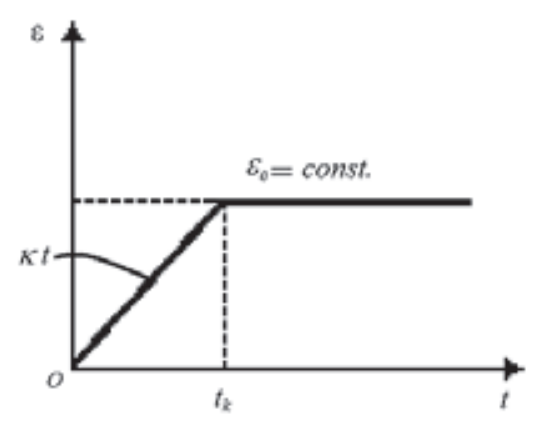

2.

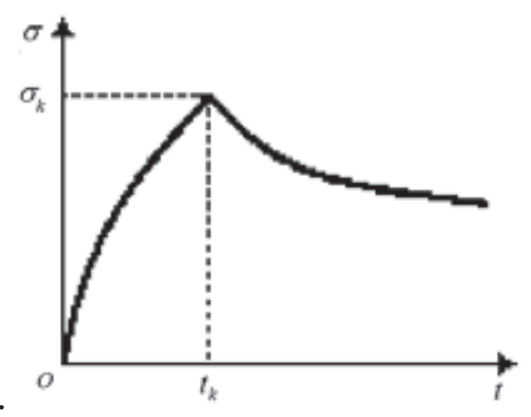

Figure 2.The ramp-and-hold strain than stress relaxation deformation pattern.

It should be noted that for the values of parameters satisfying (5) the expression (7) exhibits behavior shown in Figure 2. 
In the following section we present it for two sets of recent experimental data corresponding to sham operated and healing MCL. From the stress relaxation curves several points were chosen and then equation (7) was forced to pass through those points. Fitting procedure was performed by the Newton method.

\section{Results}

In Abramowitch et al. (2004) study the ramp-and-hold stress relaxation test was performed on the healing goat MCL and sham-operated controls. Each femur-MCL-tibia complex specimen was elongated from 0 to $3 \mathrm{~mm}$ at $10 \mathrm{~mm} / \mathrm{min}$ and held constant for a period of $60 \mathrm{~min}$. Deformation $\varepsilon_{0}=1.7 \%$ for sham operated specimens and $\varepsilon_{0}=1.8 \%$ for heeling ones, while $t_{k}=18.4 \mathrm{~s}$ for both.

The values of the four unknown constants $\alpha, E, \tau_{\varepsilon \alpha}$ and $\tau_{\sigma \alpha}$ which are describing viscoelastic properties of the medial collateral ligament are computed by use of the suggested numerical procedure. $E$ and $\sigma$ are given in $\mathrm{MPa}$, and time $t$ is given in seconds. We present obtained results for these cases - sham operated MCL:

$$
\begin{aligned}
\alpha=0.301, E & =453.188, \tau_{\varepsilon \alpha}=1.211, \\
\tau_{\sigma \alpha} & =1.439 \cdot 10^{-3}
\end{aligned}
$$

and healing MCL:

$$
\begin{gathered}
\alpha=0.345, E=128.466, \tau_{\varepsilon \alpha}=2.883, \\
\tau_{\sigma \alpha}=0.107
\end{gathered}
$$

The agreement between the model and experimental results for sham operated MCL is shown in Figure 3, where plots are shown only for $t \in[0,300]_{s}$.

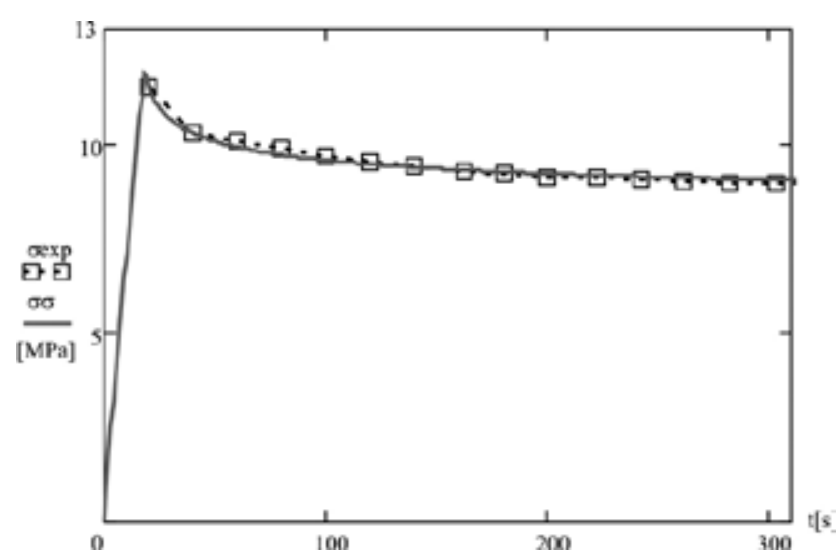

Figure 3. Measured stress relaxation experiment data for sham operated MCL (Abramowitch et al, 2004) - dashed line with marks, and predicted using fractional Zener model - solid line.

Figure 4.

The agreement between the model and experimental results for healing MCL is shown in 


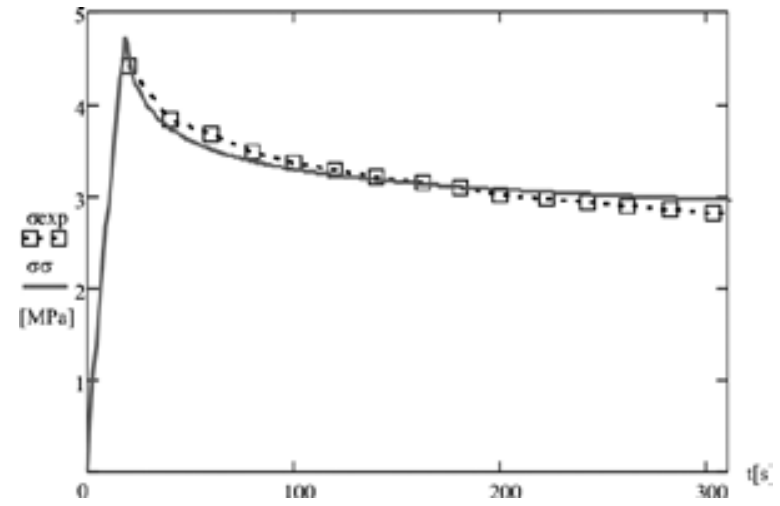

Figure 4. Measured stress relaxation experiment data for healing MCL (Abramowitch et al., 2004) - dashed line with marks, and predicted - solid line.

From Figures 3 and 4 it can be observed that the difference between the experimental data and the model is negligible.

\section{Discussion}

In this paper the rheological descriptions of the sham operated and healing MCL by means of fractional calculus were introduced. The ramp-and-hold stress relaxation experiment performed for both sham operated and healing MCL were related with the constitutive equation called fractional Zener model of viscoelastic body. First the Laplace transform was applied to that constitutive equation and the strain function corresponding to ramp and hold behaviour. Then the inversion procedure was performed. Finally the obtained solution was forced to pass trough four selected experimental points.

We found good agreement between experimental results and theoretical predictions obtained by use of the Laplace transform method applied to the fractional Zener model of viscoelastic body.

The important characteristic of the proposed model is that it is capable of predicting behavior of viscoelastic materials with significant accuracy. Also, it has only four parameters and it is simple. Four parameters could be determined for normal and pathological conditions of different intensity. Knowing the obtained constants for two different states of MCL we may predict their behavior for different load which can be done in future work. The same procedure can be applied for different states of other structures within a human body as well as for corresponding cyclic or impact loading what can be used in proper design of rehabilitation treatments.

\section{Aknowledgements}

Authors appreciate helpful discussions with Professor Zigic and Professor Spasic. 


\section{References}

Abramowitch, S. D., Woo, S. L-Y., Clineff, T. D., \& Debski, R. E. (2004). An evaluation of the quasi-linear viscoelastic properties of the healing medial collateral ligament in a goat model. Annals of Biomedical Engineering, 32(3), 329-334.

Atanackovic, T. M. (2002). A modified Zener model of viscoelastic body. Continuum Mechanics and Thermodynamics, 14, 137-148.

Bagely, R. L. \& Torvik, P. J. (1986). On the fractional calculus model of viscoelastic behavior. Journal of Rheology, 30, 133-155.

Dankuc, D. V., Kovincic, N. I., \& Spasic, D. T. (2010). A new model for middle ear structures with fractional type dissipation pattern. In: Proceedings of FDA'10. The $4^{\text {th }}$ IFAC Workshop on Fractional Differentiation and its Applications, Badajoz, Spain, October 18-20th, 2010. Article No FDA10_156. Laxenburg: by International Federation of Automatic Control.

Gorenflo, R., \& Mainardi, F. (2000). Fractional calculus: Integral and differential equations of fractional order. In A. Carpinteri \& F. Mainardi (Eds), Fractals and Fractional Calculus in Continuum Mechanics (pp. 223-276). Wien and New York: Springer-Verlag.

Grahovac, N. M., \& Zigic, M. M. (2010). Modeling of the hamstring muscle group by use of fractional derivatives. Computers and Mathematics with Applications, 59(5), 1695-1700.

Spasic, D. T., \& Charalambakis, N. C. (2002). Forced vibrations with fractional type of dissipation. In Proceedings of the International Conference on Nonsmooth/Nonconvex Mechanics with Applications in Engeneering (pp. 323-330). Thessaloniki: Aristotle University of Thessaloniki.

Woo, S. L-Y., Abramovitch, S. D., Kilger, R., \& Liang, R. (2006). Biomechanics of knee ligaments: injury, healing and repair. Journal of Biomechanics, 39, 1-20. 